\title{
Early and Specific Antibody Response to OspA in Lyme Disease
}

\author{
Steven E. Schutzer, * P. K. Coyle, ${ }^{*}$ John J. Dunn, ${ }^{5}$ Benjamin J. Luft," and Michael Brunner* \\ *Department of Medicine, Division of Allergy and Immunology, University of Medicine and Dentistry of New Jersey-New Jersey \\ Medical School; Newark, New Jersey 07103; and the "Departments of Neurology and Medicine, "Division of Infectious Diseases, State \\ University of New York, Stony Brook, Stony Brook, New York 11794; and the ${ }^{8}$ Department of Biology, Brookhaven National Laboratory, \\ Upton, New York 11973
}

\begin{abstract}
Borrelia burgdorferi ( $\mathrm{Bb})$, the cause of Lyme disease, has appeared not to evoke a detectable specific antibody response in humans until long after infection. This delayed response has been a biologic puzzle and has hampered early diagnosis. Antibody to the abundant organism-specific outer surface proteins, such as the 31-kD OspA, has rarely been detected less than 6 mo after infection. Antibody to a less organism-specific 41-kD flagellin protein, sharing common determinants with other bacteria and thus limiting its diagnostic potential, may appear after 4 to 6 wks. To investigate our hypothesis that specific antibody to OspA may actually be formed early but remain at low levels or bound in immune complexes, we analyzed serum samples from patients with concurrent erythema migrans (EM). This is the earliest sign of Lyme disease and occurs in 60-70\% of patients, generally 4-14 d after infection. We used less conventional but more sensitive methods: biotin-avidin Western blots and immune complex dissociation techniques. Antibody specificity was confirmed with recombinant OspA.

Specific complexed antibody to whole $\mathrm{Bb}$ and recombinant OspA was detected in 10 of 11 of the EM patients compared to 0 of 20 endemic area controls. IgM was the predominant isotype to OspA in these EM patients. Free IgM to OspA was found in half the EM cases. IgM to OspA was also detected in 10 of 10 European patients with EM who also had reactive $T$ cells to recombinant OspA.

In conclusion a specific antibody response to OspA occurs early in Lyme disease. This is likely to have diagnostic implications. (J. Clin. Invest. 1994. 94:454-457.) Key words: spirochete • Borrelia burgdorferi • ELISA • immune complex - Western blot
\end{abstract}

Address correspondence to Dr. Steven Schutzer, UMDNJ New Jersey Medical School, Department of Medicine, 185 South Orange Avenue, Newark, NJ 07103.

Received for publication 1 December 1993 and in revised form 22 February 1994.

1. Abbreviations used in this paper: $\mathrm{Ag}$, antigen; $\mathrm{Bb}$, Borrelia burgdorferi; EM, erythema migrans; IC, immune complex; OspA, outer surface protein A.

J. Clin. Invest.

(C) The American Society for Clinical Investigation, Inc. 0021-9738/94/07/0454/04 \$2.00

Volume 94, July 1994, 454-457

\section{Introduction}

Lyme disease is etiologically linked to the spirochete, Borrelia burgdorferi $(B b)^{1}(1,2)$. Clinical suspicion of the diagnosis has often been difficult to confirm with current serological methods (3). The human humoral response to the relatively abundant and highly specific Bb outer surface proteins such as OspA, is not usually detected until several months to years after infection $(4,5)$. Seronegative, and even some seropositive Lyme arthritis patients with a past history of erythema migrans (EM) and more than 6 mo of symptoms do not have demonstrable OspA antibody (Ab) (4) by conventional Western blots (6) or ELISA. This delayed humoral response has been puzzling and has hampered early diagnosis (7). This is particularly problematic because the early clinical marker for infection, erythema migrans (EM), which occurs on the average 4 to $14 \mathrm{~d}$ after an infected tick bite, is not observed in over one-third of cases (3). Furthermore, in many instances the rash is atypical, and a confident diagnosis of EM cannot be made. Though an $\mathrm{Ab}$ response may occur to the $41-\mathrm{kD}$ flagellin protein (8), it is usually delayed 4 to 6 wks after the infection, and its diagnostic utility is further limited because these antigenic components are commonly shared with other bacteria (4-6). Similarly, Ab may be produced to other proteins which are not unique to $\mathrm{Bb}$.

The potential to produce $\mathrm{Ab}$ to OspA early is illustrated by certain mouse infectivity models where a brisk response does occur after needle inoculation (9). We hypothesized that early after infection in humans via a tick bite, $A b$ to OspA may actually be produced at low levels not detectable by conventional methods and/or be predominantly bound in immune complexes. This follows the general observation that the normal humoral immune response to an infection involves production of $\mathrm{Ab}$ which then binds to provoking antigens (Ags) of the infectious agent (10) and shortly thereafter the $A b$ may be detectable in the free form by conventional methods. Thus the early production of some $\mathrm{Ab}$ to a specific $\mathrm{Bb}$ protein, within the first weeks of infection as opposed to months to years later, would be consistent with the biologic response to most infections. Detection of specific $\mathrm{Ab}$ has important potential for diagnosis and consequent therapy, at a time when it is most likely to be effective. In this current study, blinded analysis of serum samples obtained at the time EM was witnessed by a physician demonstrates that a humoral response to OspA frequently occurs in the first weeks of infection in Lyme disease.

\section{Methods}

Patients. All patients met the Centers for Disease Control criteria for Lyme disease as defined in reference 3 (this does not require cultures that are frequently negative). In addition for this study we required that 


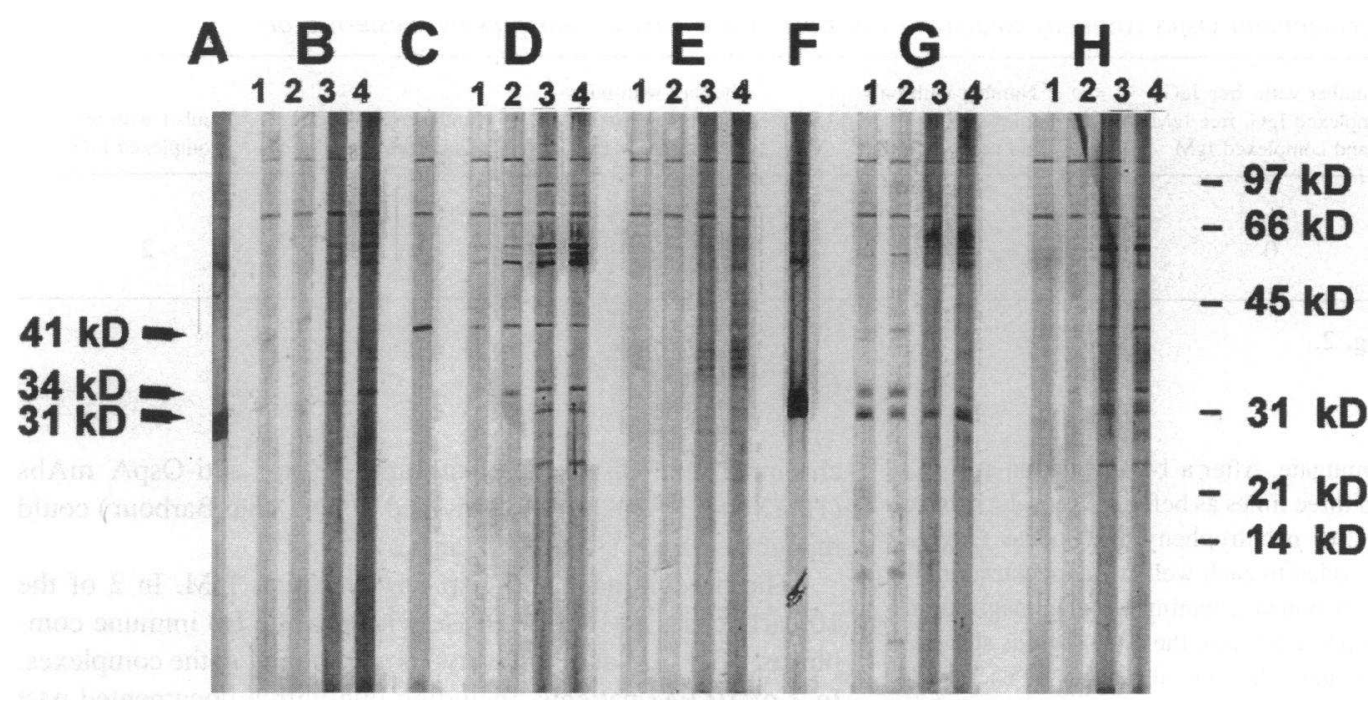

Figure 1. Representative biotin-avidin Western blot immunoreactivity of serum and serum IC Ab from early Lyme disease patients, with EM, to antigens of whole Bb B31 sonicates. Samples from chronic Lyme disease patients and mAb served as positive controls. Samples from endemic area healthy subjects served as comparative controls (Fig. 3). Human $\mathrm{Ab}$ was detected with specific conjugated goat anti-human IgM and goat anti-human IgG. The murine $\mathrm{mAb}$ was probed with a goat anti-mouse $\mathrm{Ab}$. $\mathrm{Pa}$ tients with EM are repre-

sented by groups $B, D, E$. Lyme arthritis patients are represented by $G$ and $H$. Reactivity of the patients' free and IC antibody is represented as follows: Lane 1, free IgG; lane 2, IC IgG; lane 3, free IgM; lane 4, IC IgM. Reactivity of mAb, H3TS, to $31 \mathrm{kD}$ OspA are shown in groups $A$ and $F$. Reactivity of mAb, $\mathrm{H} 9724$, to $41 \mathrm{kD}$ flagellin is shown in group $C$. Molecular weight standards are on indicated by markers on the right. Molecular weights determined by mAbs are indicated on the left.

the early cases $(n=11)$ had single or multiple EM witnessed by a physician (the lesion was photographed in eight cases), endemic area exposure, and exclusion of other diseases. Serum samples were obtained concurrent with the appearance of EM. For the later, more advanced cases $(n=10)$, requirements included in addition to exposure and exclusion of other illnesses, physician diagnosed arthritis and a seropositive Lyme disease test. Positive control sera came from previously diagnosed Lyme disease patients with active symptoms at the time of sampling. Other disease controls included a variety of infectious, immunologic, and neurologic diseases likely to have elevated circulating immune complexes. The healthy asymptomatic controls were endemic area subjects without a history of Lyme disease infection.

After the above patients from the United States were analyzed, additional sera was kindly provided by Dr. A. Krause (University of Erlangen-Nürnberg). These sera came from German patients (from reference 14) with early Lyme disease who were EM positive and were shown to have $\mathrm{T}$ cell reactivity, but not $\mathrm{Ab}$ using a conventional immunoblot, to recombinant OspA. Due to the limited volume of samples our analysis was restricted to recombinant OspA IgM biotin-avidin immunoblots for comparison.

Immune complex (IC) isolation and dissociation. Immune complexes were isolated by polyethylene glycol precipitation and dissociated as described $(11,12)$. Briefly, $0.5 \mathrm{ml}$ of the serum sample was added to an equal volume of $7 \%$ polyethylene glycol (PEG) in PBS, and incubated overnight (or alternatively $2 \mathrm{~h}$ ) at $4^{\circ} \mathrm{C}$, then centrifuged at $8,320 \mathrm{~g}$ for $15 \mathrm{~min}$. Pellets were washed twice using $3.5 \%$ PEG and resuspended in $0.5 \mathrm{ml}$ of $0.1 \mathrm{M}$ sodium borate, $\mathrm{pH} 10.2$.

Biotin-avidin Western blot. Bb (B31) sonicates (kindly provided by Dr. Marc Golightly, State University of New York, Stony Brook) or recombinant OspA were added to Laemmli gel sample reducing buffer consisting of $2 \%$ SDS, $60 \mathrm{mM}$ Tris- $\mathrm{HCl}$ ( $\mathrm{pH} 6.8$ ), $0.1 \mathrm{M} \mathrm{DTT}$, $0.005 \%$ bromphenol blue, $10 \%$ glycerol, and boiled to load each well of the preparative gel with $16 \mu \mathrm{g}$ of whole $\mathrm{Bb}$ sonicates or $1.6 \mu \mathrm{g}$ of recombinant OspA protein. Molecular weight standards were run in tandem to these samples so as to provide markers. 12\% SDS-polyacrylamide $7 \times 8 \mathrm{~cm}$ mini-gels, $0.75 \mathrm{~mm}$ thick with stack was used on the Mighty Small II system (Hoefer Sci. Instr., San Francisco, CA) for SDS-PAGE and blotting. Immunetics Miniblotter (Vector Laboratories, Inc., Burlingame, CA) was used for development. The blot was developed according to manufacturer instructions. Serum samples were added to each lane at a 1:100 dilution and the PEG immune complex precipitates at 1:10 dilution (11). Secondary Abs consisted of goat biotinylated anti-human IgG ( $\gamma$ chain specific) and biotinylated anti-human IgM ( $\mu$ chain specific), which were diluted 1:250, and biotinylated horse antimouse $\operatorname{IgG}(\mathrm{H}+\mathrm{L})$ which was diluted 1:500 (Vector Laboratories, Inc.) in TBS-T plus $1 \%$ NGS.

Recombinant OspA protein. Full length OspA lipoprotein that had been expressed in Escherichia coli and purified by Triton X-114 extraction and ion exchange chromatography (13) was used for the blots above.

ELISA for free serum antibodies to $B$. burgdorferi. Clinical serum samples were tested for anti-Bb (B31) Ab in an ELISA as described $(6,11)$. Briefly, 96-flat bottom well microtiter plates (Costar Corp., Cambridge, MA) were coated with a sonicate of Bb (B31) $(5 \mu \mathrm{g} / \mathrm{ml})$ for $18 \mathrm{~h}$ in $0.05 \mathrm{M}$ sodium carbonate $(\mathrm{pH} \mathrm{9.6)}$ and then washed three times with PBS ( $\mathrm{pH} 7.2$ ) containing $0.05 \%$ Tween 20 (PBS/Tween). Aliquots of $200 \mu \mathrm{l}$ were added to duplicate wells and incubated for 1 $\mathrm{h}$ at $37^{\circ} \mathrm{C}$. Nonspecific binding sites were then blocked by the addition to each well of $250 \mu \mathrm{l}$ solution of $1 \%$ BSA in PBS. The plates were washed with PBS-Tween. Serum was diluted 1:500. A volume of 200 $\mu \mathrm{l}$ from each sample was added to duplicate wells and incubated for 1 $h$ at $37^{\circ} \mathrm{C}$. The plates were washed with PBS-Tween. Alkaline phosphatase-conjugated goat anti-human (GAH) immunoglobulin (Ig) with specificity for heavy chain determinants was added to individually detect the anti-Bb Ig of IgM and IgG isotypes. Specific isotypes were analyzed

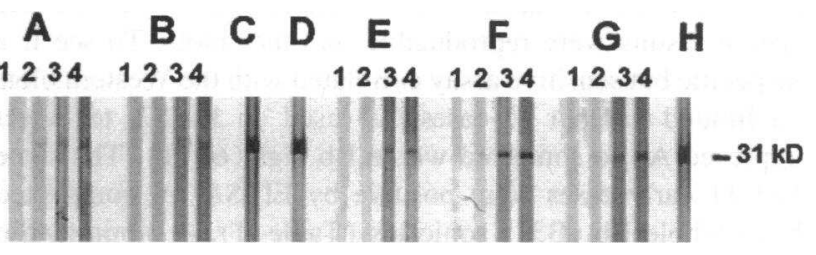

Figure 2. Representative biotin-avidin Western blot immunoreactivity of serum and serum IC Abs from patients with EM to purified recombinant OspA lipoprotein. Patients with EM are represented by groups $A$, $B, E, F, G$. Reactivity of the patients' free and IC antibody is represented as follows: lane 1, free IgG; lane 2, IC IgG; lane 3, free IgM; lane 4, IC IgM. Reactivity of mAb, H3TS, to $31 \mathrm{kD}$ OspA is shown in group $C$. Reactivity of mAb, H5332, also to OspA are shown in groups $D, H$. Molecular weight standards are indicated by markers on the right. 


\begin{tabular}{lcccc}
\hline & $\begin{array}{c}\text { Number with: free IgG, } \\
\text { complexed IgG, free IgM, } \\
\text { and complexed IgM }\end{array}$ & $\begin{array}{c}\text { Number with only: } \\
\text { complexed IgG, free IgM, } \\
\text { and complexed IgM }\end{array}$ & $\begin{array}{c}\text { Number with only: } \\
\text { free IgM and } \\
\text { complexed IgM }\end{array}$ & $\begin{array}{c}\text { Number with only: } \\
\text { complexed IgM }\end{array}$ \\
\hline $\begin{array}{l}\text { Early }(\mathrm{EM}+) \text { cases } \\
(n=11)\end{array}$ & 0 & 2 & 5 & 1 \\
\hline
\end{tabular}

* As represented partially in Fig. 2.

with the appropriate GAH Ig conjugate. After a 1-h incubation at $37^{\circ} \mathrm{C}$, the microtiter wells were washed three times as before. As a colorimetric substrate for alkaline phosphatase, $p$-Nitrophenyl phosphate (Sigma Chem. Co., St. Louis, MO) was added to each well at a concentration of $0.66 \mathrm{mg} / \mathrm{ml}$ in $0.15 \mathrm{M}$ sodium bicarbonate containing $1 \mathrm{mM}$ magnesium chloride. After incubation at room temperature the reaction was stopped with $30 \mu \mathrm{l}$ of $3 \mathrm{~N}$ sodium hydroxide. The optical density of each well was read at $405 \mathrm{~nm}$ on the ELISA reader (Bio-Tek Instr. Inc., Winooski, VT). A positive result was indicated by optical density readings greater than 3 SD above the mean of a panel of 10 healthy controls without a history of Borrelia infection. In the clinical laboratory these 10 controls represented the range of optical density readings from over 100 healthy control samples such that they yielded the same mean $+3 \mathrm{SD}$ as the larger group.

ELISA for complexed serum antibodies to $B b$. PEG precipitates were diluted 1:10 and run by ELISA (11) to separately detect IgG and IgM reactive to $\mathrm{Bb}$ Ags. IC, on ELISA, were considered to be Borrelia specific when the optical density readings of the PEG precipitate was greater than $3 \mathrm{SD}$ above the mean of at least 10 normal controls run on each plate $(6,11)$. A biotin-avidin modification with reagents similar to those of the Western blot was also used.

Statistical analysis. Fisher's exact test was used to compare the proportion of Bb-specific complexes in Lyme disease versus controls and other disease patients.

\section{Results}

Detection of $A b$ to whole $B b$ and recombinant OspA. Overall our results with biotin-avidin whole $\mathrm{Bb}$ and recombinant protein Western blots show that patients with very early Lyme disease have complexed and often free $\mathrm{Ab}$ directed against OspA.

10 of 11 early US cases had complexed $A b$ reactive to OspA, as well as to other Bb Ags, on blots to whole Bb (B31) sonicates. These 10 also had specific anti-OspA Ab on recombinant OspA blots. A representative group is shown in Fig. 1 and 2. The recombinant blots were performed to confirm that the Ab was specific for OspA rather than another protein in the same molecular weight range. The specific IgM and IgG isotypes and free/complexed $\mathrm{Ab}$ states of these samples are shown in Table I. These results were reproducible on other blots. To see if a nonspecific but sensitive assay correlated with the Western blots in a limited number of cases, we used an ELISA to detect complexed $\mathrm{Ab}$ to sonicated whole $\mathrm{Bb} \operatorname{Ags}(6,11)$. The same 10 of 11 early cases were positive by ELISA for complexed Abs to whole Bb (B31) sonicates (Table II). A biotin-avidin IgM IC ELISA was positive in 7 of 7 available EM serum samples. The controls had a mean OD of 0.08 (average ranging from 0.04 to 0.15 ) and the mean +3 SD was 0.20 . 20 healthy endemic area controls and 20 other diseased patients (infections including syphilis, autoimmune diseases such as lupus, allergic, and neurologic disorders, especially cases associated with elevated levels of immune complexes) were not reactive for either free or complexed Ab to OspA (see Fig. 3), whereas certain chronic Lyme disease patients and several anti-OspA mAbs (H3TS and H5332 kindly provided by Dr. Alan Barbour) could recognize OspA (see Fig. 1 and 2).

The predominant anti-OspA isotype was IgM. In 2 of the 10 early cases of Lyme disease with specific Bb immune complexes, both IgM and IgG isotypes were found in the complexes. In 2 of $10 \mathrm{EM}$ patients, including one with a documented past history of $\mathrm{Bb}$ infection and EM, the anti-OspA reactivity was found solely in the complexed IgG fraction. This could reflect an amnestic response. In half of the early (EM) Lyme disease patients with complexed IgM to recombinant OspA, biotin-avidin blots also detected free IgM Ab to the recombinant protein. Analysis of the German EM patients also revealed specific IgM to recombinant OspA in 10 of 10 cases (Fig. 4).

\section{Discussion}

The current studies demonstrate that OspA is recognized by the immune system, and that specific anti-OspA $\mathrm{Ab}$ can be produced early in human $\mathrm{Bb}$ infection. The predominant isotype in new acute cases was IgM, as might be expected with an early $\mathrm{Ab}$ response. In this paper we have equated "early" with the time that EM appeared or is most likely to appear.

Dissociation between $T$ cell and $B$ cell responses to antigenic components of $\mathrm{Bb}$ has been described in certain chronic seronegative patients who exhibit $\mathrm{T}$ cell reactivity to $\mathrm{Bb}$, but no detectable free $\mathrm{Ab}$ to $\mathrm{Bb}(6)$. Recently, in early Lyme disease patients, $T$ cells reactive to OspA have been identified in the absence of free Ab to OspA (14). However, complexed Ab was not investigated in these studies, nor were blots performed with biotin-avidin reagents. We point this out because both these techniques are likely to enhance the sensitivity of detection. We

Table II. Combined IgM and IgG Complexed Ab to Bb Ags in Various Patient Groups by Western Blot and ELISA

\begin{tabular}{lccc}
\hline & $\begin{array}{c}\text { Number } \\
\text { with } \\
\text { complexed } \\
\text { anti-Bb Ab }\end{array}$ & $\begin{array}{c}\text { Number } \\
\text { without } \\
\text { complexed } \\
\text { anti-Bb Ab }\end{array}$ & $P$ value \\
\hline $\begin{array}{l}\text { Early EM + Lyme disease } \\
\quad(n=11)\end{array}$ & 10 & 1 & $2 \times 10^{-7}$ \\
$\begin{array}{l}\text { Seropositive symptomatic Lyme } \\
\text { disease with arthritis } \\
(n=10)\end{array}$ & 10 & 0 & $3 \times 10^{-8}$ \\
$\begin{array}{l}\text { Other endemic area disease } \\
\text { subjects }(n=20)\end{array}$ & 0 & 20 & $<10^{-8}$ \\
$\begin{array}{c}\text { Healthy endemic area controls } \\
(n=20)\end{array}$ & 0 & 20 & - \\
\hline
\end{tabular}




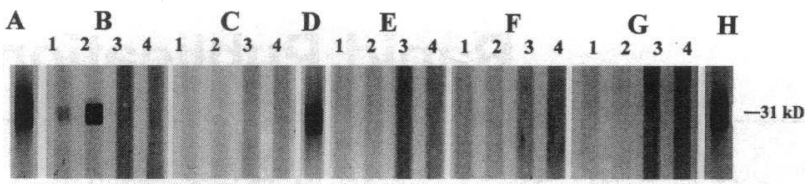

Figure 3. Representative biotin-avidin Western blot immunoreactivity of serum and serum IC Abs from control subjects to purified recombinant OspA lipoprotein. A positive Lyme disease patient is represented by group $B$. Endemic area control subjects are represented in groups $C, E$, $F, G$. Reactivity of the individuals' free and IC antibody is represented as follows: lane 1, free IgG; lane 2, IC IgG; lane 3, free IgM; lane 4, IC IgM. Reactivity of mAb, H3TS, to $31 \mathrm{kD}$ OspA is shown in groups $A, D, H$. Molecular weight is indicated by marker on the right.

were aware that the IC dissociation technique was likely to yield $\mathrm{Ab}$ that might not be detected by an assay probing only for free $\mathrm{Ab}$. With respect to techniques to develop $\mathrm{Bb} \mathrm{Ab}$ blots of known samples, preliminary experiments revealed enhanced staining with the biotin-avidin versus alkaline phosphatase reagents. This ranged from less intense staining of bands to virtual absence of staining with conventional alkaline phosphatase reagents that were otherwise seen by using the enhancing biotinavidin reagents. We reconfirmed this using recombinant OspA as the target $\mathrm{Ag}$ in the blot. The detection of complexed and free IgM to OspA in the German patients, with these methods, is in agreement with this observation. The finding of free IgM but not IgM complexed to OspA may reflect a response that has evolved to IgG. It is important to consider that factors other than technique, such as route of infection, number of infecting organisms, and the host's immune system may contribute to detection of an Ab response. Nevertheless, using an analysis of serum immune complexes and sensitive Western blot techniques, it was possible to identify in a large percentage of patients specific $\mathrm{Ab}$ to OspA within immune complexes and frequently in the free state as well.

The exact mechanisms and chronology by which $\mathrm{T}$ cells induce specific $\mathrm{Ab}$ production has yet to be delineated. The kinetics of the immune response to the various Ags of $\mathrm{Bb}$ also needs to be determined. It is still not clear why a discernible, albeit often weak, humoral response to OspA occurs early but does not fully develop to the point of detection by conventional assays until late in the disease.

Our detection of OspA Ab early in the course of infection in humans agrees with certain animal infectivity models that are also capable of humoral immune recognition of OspA. The precise parallels to animal models require further study with particular consideration to the route of infection and the method

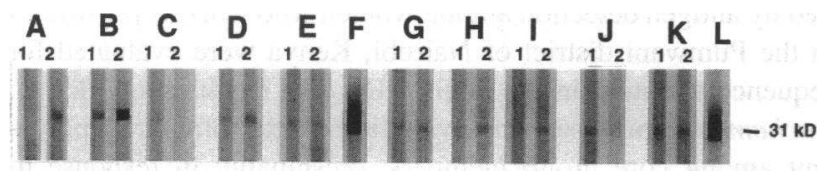

Figure 4. Representative biotin-avidin Western blot IgM immunoreactivity to purified recombinant OspA lipoprotein of serum and serum IC Abs from patients with EM and T cell reactivity to OspA. Patients with $\mathrm{EM}$ and $\mathrm{T}$ cell reactivity to OspA are represented by groups $A, B, C$, $D, E, G, H, I, J, K$. Reactivity of the patients' free and IC antibody is represented as follows: lane 1, free IgM; lane 2, IC IgM. Reactivity of $\mathrm{mAb}, \mathrm{H} 5332$, to OspA is shown in groups $F, L$. Molecular weight standard is indicated by marker on the right. of analysis. The animals mount a brisk and early response to OspA when needle-inoculated with $\mathrm{Bb}(9,15)$. Yet when infected by ticks, they seem not to develop this specific response until very late in the infection (15), as analyzed by conventional assays. However, in these models, early serum samples have not yet been examined with the present techniques.

In humans, the ability to detect $A b$ to OspA in the early weeks resolves part of a biologic puzzle. Detection of specific $\mathrm{Ab}$ has important potential to support a clinical diagnosis in this illness, especially at the outset, when therapy is most likely to be effective, and can be a diagnostic aid in patients who were bitten by ticks but have not developed the typical rash (16).

\section{Acknowledgments}

The authors thank Dr. Andreas Krause for supplying additional samples.

This work was supported in part by grants from National Institutes of Health (AR-41518, AI-324501, AR-40470, and AI-31561), Centers for Disease Control (U50/CCU206582 and U50CCU206608), and New Jersey Good Sam Club.

\section{References}

1. Steere, A. C., R. L. Grodzicki, A. N. Kornblatt, J. E. Craft, A. G. Barbour, W. Burgdorfer, G. P. Schmid, E. Johnson, and S. E. Malewista. 1983. The spirochetal etiology of Lyme disease. N. Engl. J. Med. 308:733-740.

2. Benach, J. L., E. M. Bosler, J. P. Hanrahan. 1983. Spirochetes isolated from the blood of two patients with Lyme disease. N. Engl. J. Med. 308:740742.

3. Rahn, D. W., and S. E. Malewista. 1990. Lyme disease: Recommendations for diagnosis and treatment. Ann. Intern. Med. 114:472-481.

4. Craft, J. E., D. K. Fischer, G. T. Shimamoto, and A. C. Steere. 1986. Antigens of Borrelia burgdorferi recognized during Lyme disease. Appearance of a new immunoglobulin $M$ response and expansion of the immunoglobulin $G$ response late in the illness. J. Clin. Invest. 78:934-939.

5. Golightly, M. G., and A. L. Viciana. 1992. ELISA and immunoblots in the diagnosis of Lyme borreliosis. Curr. Commun. Cell and Mol. Biol. 6:283-297.

6. Dattwyler, R. J., D. J. Volkman, B. J. Luft, J. J. Halperin, T. J. Thomas, and M. G. Golightly. 1988. Seronegative Lyme disease. Dissociation of specific T- and B-lymphocyte responses to Borrelia burgdorferi. N. Engl. J. Med. 319:1441-1446.

7. Barbour, A. G. 1989. The diagnosis of Lyme Disease: Rewards and perils. Ann. Intern. Med. 110:501-502.

8. Barbour, A. G., S. F. Hayes, R. A. Heiland, M. E. Schrumpf, and S. L. Tessier. 1986. A Borrelia-specific monoclonal antibody binds to a flagellar epitope. Infect. Immun. 52:549-554.

9. Fikrig, E., S. W. Barthold, F. S. Kantor, and R. A. Flavell. 1990. Protection of mice by against the Lyme disease agent by immunizing with recombinant OspA. Science (Wash. DC). 250:553-556.

10. Theofilopoulos, A. N., and F. J. Dixon. 1980. Immune complexes in human diseases. Am. J. Pathol. 100:531-591.

11. Schutzer, S. E., P. K. Coyle, A. L. Belman, M. G. Golightly, and J. Drulle. 1990. Sequestration of antibody to Borrelia burgdorferi in immune complexes in seronegative Lyme disease. Lancet. 335:312-315.

12. Coyle, P. K., S. E. Schutzer, A. L. Belman, L. B. Krupp, and M. G. Golightly. 1990. Cerebrospinal fluid immune complexes in patients exposed to Borrelia burgdorferi: detection of Borrelia specific and non-specific complexes. Ann. Neurol. 28:739-744.

13. Dunn, J. J., B. A. Lade, and A. G. Barbour. 1990. Outer surface protein A (OspA) from the Lyme disease spirochete, Borrelia burgdorferi: high level expression and purification of a soluble recombinant form of OspA. Protein Expression Purif. 1:159-168.

14. Krause, A., G. R. Burmester, A. Rensing, C. Schoerner, U. E. Schaible, M. M. Simon, P. Herzer, M. D. Kramer, and R. Wallich. 1992. Cellular immune reactivity to recombinant OspA and flagellin from Borrelia burgdorferi in patients with Lyme borreliosis. Complexity of humoral and cellular responses. J. Clin. Invest. 90:1077-1084.

15. Roehrig, J. T., J. Piesman, A. R. Hunt, M. G. Keen, C. M. Happ, and B. J. B. Johnson. 1992. The specificity of the hamster immune response to Borrelia burgdorferi proteins depends upon source of antigen and route of infection. Fifth International Conference on Lyme Borreliosis Arlington, VA. June 1992. A36. (Abstr.).

16. Kassirer, J. P. 1992. Is a tick's bite worse than its bite? Formulating an answer with decision analysis. N. Engl. J. Med. 327:562-563. 